\title{
Social Public Security Management in Intelligent City Based on Fast Big Data Analysis
}

\author{
Tong Zhu ${ }^{1,2, \text { a }}$ \\ ${ }^{1}$ College of Electronic and Information Engineering, Tongji University, 4800 Cao'an Highway, \\ Shanghai, China, 201804 \\ ${ }^{2}$ Anti-counterfeiting Technology Division, The Third Research Institute of The Ministry of Public, \\ Security of The P. R. C., Bisheng Road 339, Shanghai, China, 201204 \\ afw339zt@126.com
}

Keywords: Intelligent City;Grid management Fast Big Data Analysis; Social Public Security Management; Data Hierarchy and Knowledge Map Model;

\begin{abstract}
Social public security management is an important component in the current construction of intelligent cities in China. There have been scale construction of information system for social public security management, which has been widely applied, generating abundant information data in the city's "grid" management process. However, in the actual construction process of intelligent city, due to the data conversion between various information systems and various restrictions for interconnection, further promotion and use of the social public security management has been seriously affected. In some big cities, every day there are tens of millions of new sensors collecting big data and store it for a long time. And it is difficult to meet the requirement of real-time management through the exchange between traditional information systems, urgently needing intelligent analysis and process to give full play to the application value. Taking solving "noise disturbance" as an example, introduces that the "Fast Big Data Analysis" method will dynamically realize the perception of social public security situation, performs predictive judgment and management guidance based on city-level data resource sharing and urban "grid" management, to effectively improve the overall management performance of the social public security management in the intelligent city.
\end{abstract}

\section{Introduction}

As the two major pillars of the science system, the humanities and natural sciences have developed almost parallel in the very different paradigms since their inception. Nowadays, the big data provides a new perspective for humanities, reforms the traditional paradigms of humanities, blurs the boundaries between the humanities and the natural sciences, consequently the humanities and natural sciences go together, showing the trend of integration of humanities and natural sciences ${ }^{[1]}$. Over the past half century, with the full integration of computer science into social life, and information explosion has been accumulated to an extent starting to cause reform. It not only makes the world filled with a lot more information than ever before, and the growth speed is also accelerating. Also, changes in the total amount of information have caused changes in the form of information- quantitative change to qualitative change. At present, this concept has almost been applied to almost every field that humans are devoted to developing ${ }^{[2]}$. After accumulation of a lot of data, technologies of data mining, machine learning are needed to find out the models that have significance on the business from the mass data. For example, if the power consumption data collected and stored in the smart meter installed in various families and enterprises is combined with the external factors of season, weather information, the mode "power consumption over 95\% of the power supply capacity" can be mastered ${ }^{[3]}$.

The latest development under current era, is the quick improvement of computing capacity and expansion of information technology to every field of the society. The cyberspace has started to dominate physical space and has begun to merge with physical spaces in large city centers. Human 
activities have been more and more "data-based" and become part of a "measurable, analyzable" system ${ }^{[4]}$. In this new era, machine intelligence will be reflected in all aspects of human life and work, such as transportation, medical, education, appliances, public security, machine production and so on, there will be a lot of automation in each field, but in almost each of these fields, finally they will intersect at one point, which is city. Proposal of the concept of intelligent city, to a certain extent, can be regarded as the first move of human towards the intelligence era. The intelligent city means to progress towards efficient development and intelligence, which hits the pain spot of government officials and urban managers in the world, thus widely accepted by governments of all nations ${ }^{[5]}$. Arrival of the big data era has a significant impact on the extensive public security management idea that has been formed in China for a long time. Data has been playing a more and more important role in the public security management, a "lifeline" and "blood transfusion tube" of strategy and decision making.

\section{Building city-level data resource sharing model based on grid management}

In the intelligent city management in China, application of grid management has become a trend in the city core area with a large population density in the large and medium-sized cities. The grid management divides the city administration jurisdiction into unit grids for monitoring and fine operation and management. Administrators under this jurisdiction carries out the all-region management model by grade and by layer, to better integrate various approaches, share resources, transforming the urbanization management from extensive to fine, concrete and data-based, to realize the efficient management and precise and orderly positioning of each part of the city ${ }^{[7]}$. The social public security management is one of the key contents in the grid management of the city core area. The city core area can generally be divided into the three levels of administrative area, streets and grids, shown as in Fig.1.

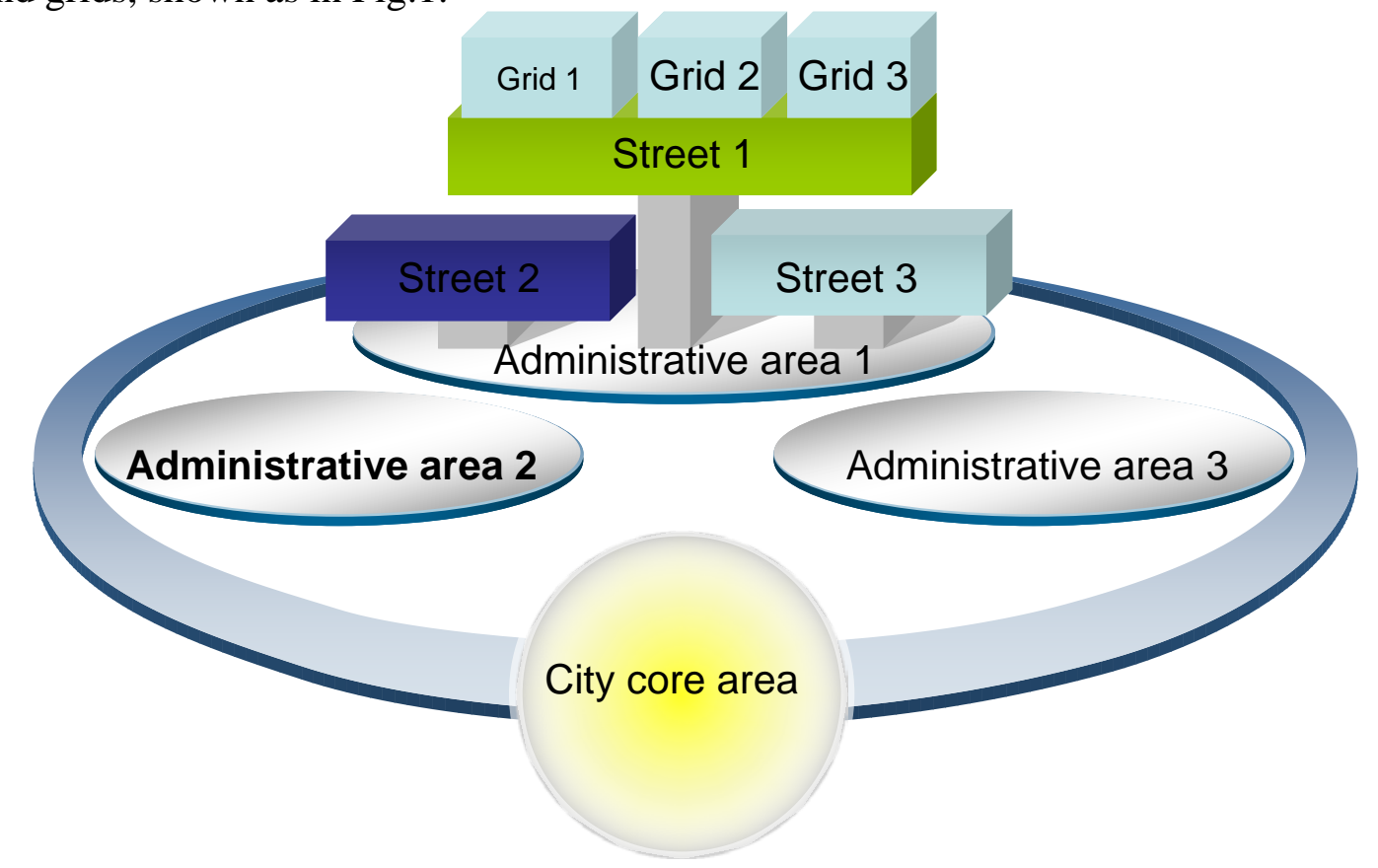

Fig.1 City core area management hierarchy model

In the city-level management system, all kinds of information consist of raw data, historical data, exported data and conclusion data, natural extension process of data separation caused by differences in data sources is shown as in Fig.2. 


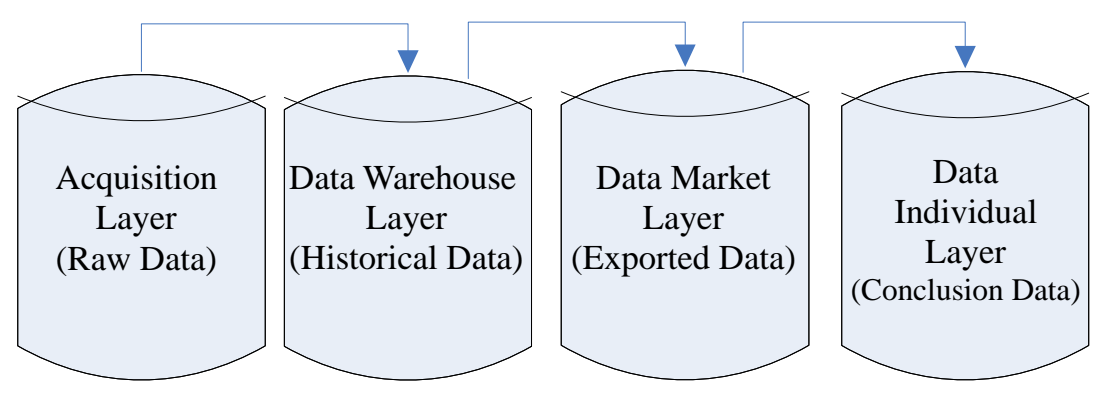

Fig.2 Data constitution of the big data set

Through the four categories of acquisition layer, data warehouse layer, data market layer, data individual layer, thus forming the fundamental big data set of social public security management. Where, the acquisition layer data includes only raw data for application, mainly serving the high-performance transaction processing. The data warehouse stores the integrated historical data that cannot be updated, moreover, it can also store some exported data. To meet the final management requirements, the data market layer is for the exported data for data exchange. Most heuristic analysis is completed in the individual layers of data ${ }^{[8]}$.

In the intelligent city planning, the data acquisition layer can be built, operated and maintained on the local grid level, while the data warehouse can be built, operated and maintained on the city level, and the data market layer can be built, operated and maintained on both the administrative area and street levels, while the data individual layer can be built, operated and maintained on the city, administrative area, street, grid respectively. Grid-based management and city-level data resource sharing model can thus be built, shown as in Fig.3:

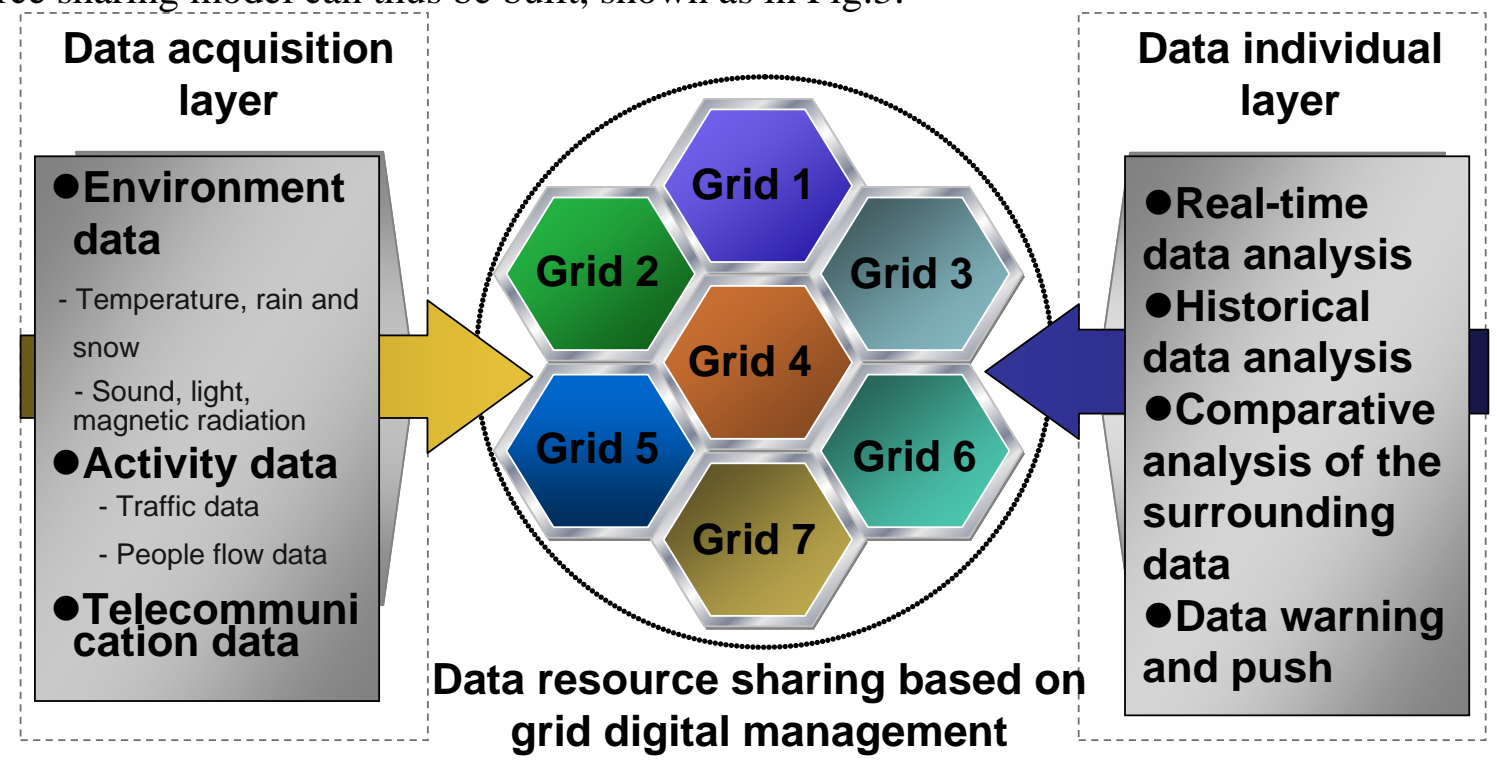

Fig.3 Data resource sharing model based on grid digital management

\section{Case analysis for the fast big data analysis for social public security management}

On the basis of building the data resource sharing model based on grid management, through fast big data analysis, we can realize the application for social public security management. Taking "noise control" as an example, we can set up main entity components with sound intensity (sound sensor equipment) and traffic/people flow status (video acquisition and analysis equipment) as strong coupling, namely each group of sensors consists of a set of sound sensor equipment and a set of video acquisition and analysis equipment; temperature, humidity, surrounding factory/commerce 
status information, air quality information, climate information and other external and previous historical record information as the loose coupling components, with location and time as the boundary to correlate the semantic relationship between various entities, and the obtained knowledge map model is shown as in Fig.4:

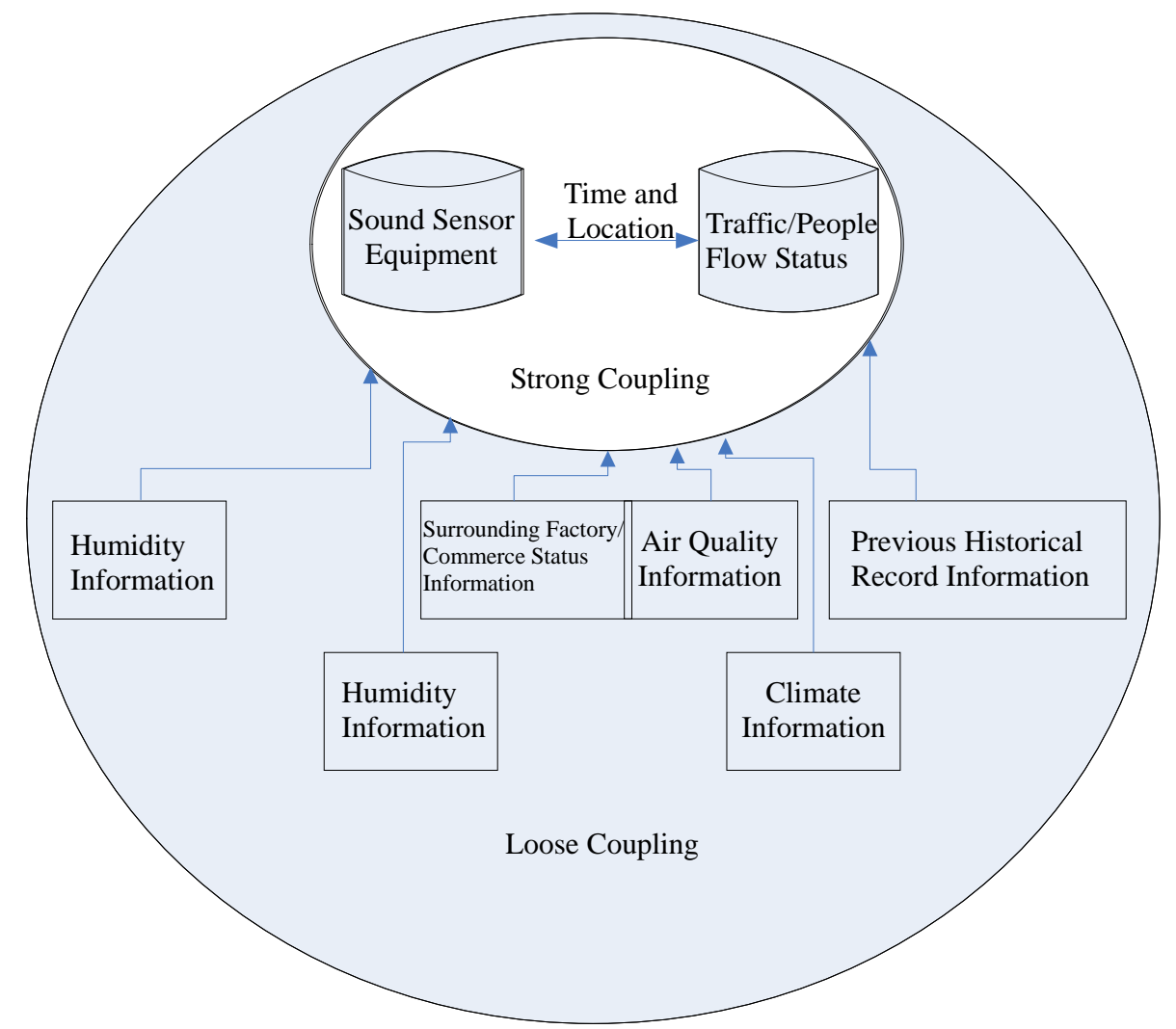

Fig.4 Knowledge map model with sound intensity (sound sensor equipment) and traffic/people flow status (video acquisition and analysis equipment) as strong coupling

Based on completing the fundamental knowledge map model and acquiring knowledge according to the knowledge acquisition process, through the three-element storage and maintenance; addition of relational timing characteristics of two methods: extensive labeling and precise labeling; as well as the subsequent relationship credibility calculation, finally the semantic link model with the "sound intensity" and "traffic/people flow status" as the strong coupling condition under the big data environment. When condition of the focus changes, we can extract elements of the loose coupling entity components to add or replace elements in the previous strong coupling, to form new semantic link models.

\section{To realize the noise prediction and management with the fast big data analysis method}

In practical applications, the data collection of front-end multi-sensor devices is usually large, far exceeding the processing power of existing traditional computing technologies and information systems. Therefore, it has been an urgent need of the real world to seek effective big data processing technologies, methods and approaches.

In building the "noise control" system, on the basis of knowledge map pretreatment, the big data performs correlation analysis for various factors with the information dynamically input by each group of sensors, to carry out the fast big data analysis, through which the "noise prediction" results can be obtained. The specific process can be divided into three stages.

Step 1, to collect data on the acquisition layer. In the example of "noise control", node of each group of sensors is the node that supports Hadoop data storage and parallel computing, and statistical analysis is carried out by $\mathrm{R}$ for the numerical information collected by the sensor, 
including the instantaneous sound value, sound change value per unit time collected by the sound sensor, and the traffic/people flow status information collected by the video acquisition and analysis equipment. When the collected data is at the warning threshold of the node, the data is preferentially aggregated to the data warehouse layer and processed in the second step.

Step 2, the data market layer further collects the required relevant data, which is gathered to the data warehouse layer. Including the temperature information, humidity information, surrounding factory/commerce status information, air quality information, climate status information and other external environment and previous historical record information; numerical information collected by the sensor at the adjacent node around the current node. The Hadoop computer cluster technology is applied to process the big data obtained at each group of acquisition sensor nodes and data market layer, and the $\mathrm{R}$ language (hereafter referred to as R) is applied for data statistics.

Step 3, final data analysis and processing on the data individual layer, on the model building method, the classic Genetic Algorithm can be applied as reference ${ }^{[10]}$, to analyze and judge the noise trend. And the analysis results are to be displayed to relevant customers.

Through the above-mentioned fast big data analysis, the "noise prediction" results can be obtained, and the noise will be treated and controlled through the grid management model.

\section{Conclusion}

With rapid development of big data, cloud computing, sensor technologies, it can provide strong support for the improvement of the overall management performance of social public security management, and it will definitely have a profound impact on the construction and management of intelligent city in the future.

\section{References}

[1] Erez Aiden, Jean-Baptiste Michel. Uncharted-Big Data as a Lens on Human Culture. Wang Tongtong, Shen Huawei, Cheng Xueqi (translation), Zhejiang, Zhejiang People's Publishing House, 2015.9.

[2] Viktor Mayer-Schonberger, Kenneth Cukier. Big Data-A Revolution That Will Transform How We Live,Work,And Think. Sheng Yangyan, Zhou Tao (translation), Zhejiang, Zhejiang People’s Publishing House, 2013.3.

[3] Yoshida Masaki. The impact of big data. Zhou Ziheng (translation), Beijing, Posts\& Telecom Press, 2013.6.

[4] Henry Kissinger. World Order. Hu Liping, Lin Hua, Cao Aiju (translation), Beijing, CITIC Press Group, 2015.8.

[5] Tu Zipei. Big Data-History,Reality and Future, Beijing, CITIC Press Group, 2014.5.

[6] Chen Tan et al. National governance in the era of big data. Beijing: China social sciences press, 2015.1.

[7] Zhu Sijing. Problems in the grid management in the cities in China and the countermeasures[J]. Economic Research Guide. 2016(17):160-161.

[8] Inmon.W.H. Wang Zhihai et al (translation). Data warehouse (4th edition of the original book). Beijing: China Machine Press, 2006.8

[9] Huang Yihua, Miao Kaixiang et al. Deep understanding of big data: big data processing and programming practice. Beijing: China Machine Press, 2015.6.

[10]Melanie Mitchell. Complexity: A Guided Tour, First Edition. Tang Lu (translation). Hunan: Hunan Science \& Technology Press, 2011. 6. 